\title{
KINETICS OF THERMAL EXCITATION OF A MOLECULE IN A CONDENSED MEDIUM
}

\author{
V.I. TESLENKO, ${ }^{1}$ D.Y. IATSENKO ${ }^{2}$ \\ ${ }^{1}$ Bogolyubov Institute for Theoretical Physics, Nat. Acad. of Sci. of Ukraine \\ (14b, Metrologichna Str., Kyiv 03143, Ukraine; e-mail: vtes@bitp. kiev. ua) \\ ${ }^{2}$ National Taras Shevchenko University of Kyiv \\ (2, Prosp. Glushkova, Kyiv 03022, Ukraine)
}

12] involved in fluctuation-dissipation relations [9-11,

A microscopic model of thermal excitation of the vibrational ground state of a molecule interacting with a condensed medium is developed. The master equation for the evolution of occupancies of vibrational levels is derived. The rate constant limiting the process of molecular thermal excitation is determined analytically. It is shown that while this quantity is independent of the temperature at low temperatures, by coinciding with a rate speed limit for quantum transitions due to the uncertainty principle, it rises rather linearly with the temperature at high temperatures according to the Einstein's relation for adiabatic transitions.

\section{Introduction}

Considering the kinetic and dynamic processes in different atomic and molecular nanostructures, for example, in molecular impurity centers, metal or semiconductor nanoparticles, biological macromolecules, etc., requires the knowledge of microscopic mechanisms for the vibrational relaxation of a system at limiting stages of its evolution. In recent years, this problem became very important, particularly in the context of the invention and implications of superfast heat generation in the close vicinity of gold nanoparticles under impulsive irradiation $[1,2]$. From the physical point of view, such process is analogous to the instantaneous formation of a point source of heat energy for molecules [3] followed by their thermal excitation and the subsequent transition to higher vibrational levels. Therefore, in the frame of a microscopic approach, one has to solve the problem of the impact of thermodynamic fluctuations in a condensed medium at a certain temperature on the process of vibrational relaxation of a molecule that was initially in the ground vibrational state.

Many attempts were made to develop the kinetic microscopic models of vibrational relaxation in molecules [4-9]. However, the attention was mainly paid to the analysis of intra- and intermolecular anharmonicity $[5,6,9,10]$ and the role of spectral factors $[9-$
13]. At the same time, some specific types of random motions, particularly thermal noise (that is nonlinear over the molecular degrees of freedom), do not obey the fluctuation-dissipation relations in the general case [14]. To approach such types of motion, we use a concept of generalized open quantum system that interacts with a heat bath $[15,16]$. Within this framework, one can consider both the bilinear intermolecular anharmonicity (that, according to the fluctuationdissipation theorem, causes one-phonon relaxation transitions between molecular vibrational levels) and thermal noise (that randomly shifts these levels). We construct a microscopic model for the thermal excitation of the molecular vibrational ground state and derive the master equation for level occupancies. Moreover, we determine the rate constant of thermal excitation analytically and show that, in the general case, this quantity exhibits the simple temperature dependence $\sim\left\{2 \omega_{0}+\left(2 \omega_{c} / \pi\right) \exp \left(\hbar \omega_{c} / 2 k T\right)\left[\exp \left(\hbar \omega_{c} / k T\right)-1\right]^{-1}\right\}$, where $\hbar$ and $k$ denote the Planck and Boltzmann constants, respectively, while the frequencies of vibrations in a given molecule $\omega_{0}$ and the molecular environment $\omega_{c}$ are determined only by structural factors and do not depend on the temperature $T$.

\section{Hamiltonian of a System}

We define the ground vibrational state as a state with the highest kinetic energy $K(q)=-\frac{\hbar^{2}}{2 m} \frac{\partial^{2}}{\partial q^{2}}$ and lowest potential energy. The latter in the harmonic approximation takes the form $U(q,\{Q\})=$ $U_{0}+\left.(1 / 2)\left\{\partial^{2} U(q,\{Q\}) / \partial q^{2}\right\}\right|_{q=q_{0}}\left(q-q_{0}\right)^{2}$, where $U_{0} \equiv$ $\left.U(q,\{Q\})\right|_{q=q_{0}}=0$. Here, $q$ and $q_{0}$ denote the normal nuclear coordinate of a given molecule (for simplicity, we consider only one such coordinate with reduced mass $m$ ) and its equilibrium value found from the condition $\left.\left\{\frac{\partial}{\partial q} U(q,\{Q\})\right\}\right|_{q=q_{0}}=0$, respectively. Potential energy depends also on displacements of the 
normal nuclear coordinates of molecules in the environment $\{Q\}$ from their equilibrium positions $\left\{Q_{0}\right\}$, that is necessary for the occurrence of relaxation transitions between molecular vibrational levels. The interaction part of the expansion of the potential energy in mutual displacements $\left(q-q_{0}\right)\left(\{Q\}-\left\{Q_{0}\right\}\right)$ responsible for such transitions appears in the form $V_{\text {rel }}=$ $\left.\left\{\frac{\partial^{2}}{\partial q \partial\{Q\}} U(q,\{Q\})\right\}\right|_{q=q_{0},\{Q\}=\left\{Q_{0}\right\}}\left(q-q_{0}\right)\left(\{Q\}-\left\{Q_{0}\right\}\right)[5$, 6]. In this expansion, the term $\sim\left(q-q_{0}\right)^{3}$ is excluded, which may be valid when the center of molecular vibrations coincides with the center of inversion. We note that there are no terms in $V_{\text {rel }}$ that do not make a key contribution to the kinetics of relaxation transitions on the limiting stage of establishment of the final equilibrium, as well as terms that can be taken into account by the renormalization of the Hamiltonian of the environment $H(\{Q\})[6]$ (i.e., terms $\sim\left(q-q_{0}\right)^{2}\left(\{Q\}-\left\{Q_{0}\right\}\right) ;\left(q-q_{0}\right)^{4}$ and $\left(\{Q\}-\left\{Q_{0}\right\}\right) ;\left(q-q_{0}\right)\left(\{Q\}-\left\{Q_{0}\right\}\right)^{2}$, respectively). Thus, in the representation of oscillator wave functions $|n\rangle$ (where $n=0,1,2, \ldots$ is the number of the vibrational state), the respective Hamiltonian of the entire system (molecule + environment + interaction) takes the form

$H(t)=H_{0}(t)+H_{T}+V_{\text {rel }}$.

In this expression,

$H_{0}(t)=\sum_{n} \hbar \omega(t)(n+1 / 2)|n\rangle\langle n|$

represents the main Hamiltonian of a molecule under consideration, which is a stochastic operator depending on the random realizations of molecular movement in the environment along the quasiclassical nuclear coordinates $\{Q(t)\}$ involved in the expression for frequency of molecular vibrations:

$[\omega(t)]^{2} \equiv[\omega(Q(t))]^{2}=\left.\frac{1}{m}\left\{\frac{\partial^{2}}{\partial q^{2}} U(q, Q(t))\right\}\right|_{q=q_{0}}$.

In addition, in Eq. (1),

$H_{T}=\sum_{\lambda} \hbar \Omega_{\lambda}\left(b_{\lambda}^{+} b_{\lambda}+1 / 2\right)$

is the Hamiltonian of a condensed medium which can be represented in the harmonic approximation as a heat bath having an infinite set of the normal vibrations (phonons) with frequencies $\Omega_{\lambda}$. The quantities $b_{\lambda}^{+}$and $b_{\lambda}$ refer to the operators of creation and annihilation of the respective $\lambda$ th mode. The last term in (1),

$V_{\mathrm{rel}}=\sum_{n \lambda} \hbar \chi_{\lambda} \sqrt{n+1}(|n\rangle\langle n+1|+| n+1\rangle\langle n|)\left(b_{\lambda}^{+}+b_{\lambda}\right)$, represents the operator of relaxation transitions in the one-phonon approximation that describes transitions between the nearest vibrational levels. The corresponding parameter of bilinear intermolecular anharmonicity

$\chi_{\lambda}=\sqrt{\xi_{\lambda} \varpi_{0} \Omega_{\lambda}}$

characterizes the dependence of relaxation transitions at both the frequencies of normal vibrations in a heat bath $\Omega_{\lambda}$ and the shifted frequency of molecular vibrations $\left.\varpi_{0} \equiv \omega(Q(t))\right|_{Q=Q_{0}}$. This frequency shift appears due to the interaction of a molecule with a random mean field of thermal motions in the environment. The respective nonshifted frequency is $\omega_{0} \equiv \varpi_{0}-\sigma$, where $\omega_{0}^{2}=\left.\frac{1}{m}\left\{\frac{\partial^{2}}{\partial q^{2}} U^{(0)}(q)\right\}\right|_{q=q_{0}}$ with $U^{(0)}(q)$ being the timeindependent part of the potential energy. The latter can be found from the condition $\left.\left\{\frac{\partial}{\partial\{Q\}} U(q,\{Q\})\right\}\right|_{q=q_{0}}=0$. The corresponding standard mean field deviation is

$\hbar \sigma=\frac{\hbar}{\sqrt{m}}\left\{\sqrt{\left.\left[\frac{\partial^{2}}{\partial q^{2}} U(q,\{Q(t)\})\right]\right|_{q=q_{0},\{Q\}=\left\{Q_{0}\right\}}-}\right.$

$\left.-\sqrt{\left.\left[\frac{\partial^{2}}{\partial q^{2}} U^{(0)}(q)\right]\right|_{q=q_{0}}}\right\}$

while

$\xi_{\lambda}=$

$=\frac{1}{4} \frac{\left(\left.\left\{\partial^{2} U(q,\{Q\}) / \partial q \partial Q_{\lambda}\right\}\right|_{q=q_{0},\{Q\}=\left\{Q_{0}\right\}}\right)^{2}}{\left.\left.\left\{\partial^{2} U(q,\{Q\}) / \partial q^{2}\right\}\right|_{q=q_{0}}\left\{\partial^{2} H(\{Q\}) / \partial Q_{\lambda}^{2}\right\}\right|_{\{Q\}=\left\{Q_{0}\right\}}}$,

defines the so-called parameter of nonadiabaticity. The latter couples the molecular vibrations with those of the environment (in the case of adiabatic coupling, the molecular coordinate must coincide with one of the normal coordinates $q=Q_{\lambda^{\prime}}$, and the respective parameter is thus $\xi_{\lambda^{\prime}}=1$ ).

From the general form of Hamiltonian (1) of the system, one can see that the problem of the thermal excitation of a molecule in a condensed medium is reduced to the problem of description of the evolution of the harmonic quantum system (2) having the randomly alternated energy levels (3). By the energy conservation law (principle of microscopic equilibrium), a molecule exhibits the persistent exchange of phonons with the heat bath (4) through the one-phonon mechanism (5). Thus, for the derivation of a master equation, it is reasonable to use the approach that has been recently developed in papers $[15,16]$ just for such types of open quantum systems. 


\section{Master Equation}

To perform an analysis of the behavior of a molecule during the process of its thermal excitation, the master equation for the evolution of observable state occupancies $\gamma_{n}(t)$ is required. Since the frequency of transitions between the molecular energy levels is the stochastic quantity, the observable state occupancies appear to be averaged over random realizations of shifts of the energy levels: $\gamma_{n}(t)=\left\langle\left\langle\Gamma_{n}(t)\right\rangle\right\rangle$, where $\langle\langle\ldots\rangle\rangle$ denotes the averaging over random shifts, while $\Gamma_{n}(t)=\langle n|\rho(t)| n\rangle$ represent the nonaveraged state occupancies. The molecular nonequilibrium density matrix $\rho(t)=\operatorname{tr}_{T} \rho_{S+T}(t)$ is determined as a trace of the nonequilibrium density matrix of the entire system $\rho_{S+T}(t)$ over the thermal bath states $\left(t r_{T}\right)$. In turn, the evolution of the entire system is governed by the Liouville equation

$\dot{\rho}_{S+T}(t)=-i \mathcal{L}(t) \rho_{S+T}(t)$,

where $\mathcal{L}(t)=(1 / \hbar)[H(t), \ldots]$ is the Liouville stochastic superoperator that acts in the space of both dynamic and stochastic variables of Hamiltonian (1) of the entire system. Expanding (7) into diagonal and offdiagonal components and solving the obtained equations for the diagonal component of the molecular density ma$\operatorname{trix} \rho_{d}(t) \equiv\left(t_{T} \rho_{S+T}(t)\right)_{\text {diag }}=\sum_{n} \Gamma_{n}(t)|n\rangle\langle n|$ yields $\dot{\rho}_{d}(t)=-\int_{0}^{t} d t^{\prime} \operatorname{tr}_{T}\left[\hat{T}_{d} \mathcal{L}_{V} S\left(t, t^{\prime}\right) \mathcal{L}_{V} \hat{T}_{d} \rho_{S+T}\left(t^{\prime}\right)\right]$, where $\mathcal{L}_{V} \equiv(1 / \hbar)\left[V_{\text {rel }}, \ldots\right]$ and $S\left(t, t^{\prime}\right)=e^{-i \int_{t^{\prime}}^{t} d \tau\left(1-\hat{T}_{d}\right) \mathcal{L}(\tau)}$ with $\hat{T}_{d}$ being the projection superoperator that translates any operator into its diagonal component.

In many practical cases, it is common that a characteristic time of transition processes $\tau_{t r}$ significantly exceeds the characteristic time of thermal relaxation $\tau_{T}$. This allows one to use a factorization $\hat{T}_{d} \rho_{S+T}(t)=\rho_{d}(t) \rho_{T}$, where $\rho_{T}=\exp \left(-H_{T} / k T\right) / t r_{T} \exp \left(-H_{T} / k T\right)$ is the bath equilibrium density matrix. Thus, in the Born approximation for the Liouville equation, one arrives at the coarse-grained master equation

$\dot{\rho}_{d}(t)=-\frac{1}{\hbar^{2}} \int_{0}^{t} d t^{\prime} \operatorname{tr}_{T}\left\{\hat{T}_{d}\left[V_{\mathrm{rel}}, U\left(t, t^{\prime}\right)\left[V_{\mathrm{rel}}, \rho_{d}\left(t^{\prime}\right) \rho_{T}\right] \times\right.\right.$

$\left.\left.\times U^{+}\left(t, t^{\prime}\right)\right]\right\}$,

where $U\left(t, t^{\prime}\right)=\hat{T} \exp \left[-(i / \hbar) \int_{t^{\prime}}^{t} d \tau\left(H_{0}(\tau)+H_{T}\right)\right]$, and $\hat{T}$ is the Dyson's chronological operator.

Taking (8) into account and using the exact form of Hamiltonian (2),(4), and (5), one can derive the equation for nonaveraged state occupancies $\Gamma_{n}(t)$ :

$$
\begin{aligned}
& \dot{\Gamma}_{n}(t)=\int_{0}^{t} d t^{\prime}\left\{\mathcal{G}_{n+1 n}\left(t, t^{\prime}\right) \Gamma_{n+1}\left(t^{\prime}\right)+\mathcal{G}_{n-1 n}\left(t, t^{\prime}\right) \Gamma_{n-1}\left(t^{\prime}\right)-\right. \\
& \left.-\left[\mathcal{G}_{n n+1}\left(t, t^{\prime}\right)+\mathcal{G}_{n n-1}\left(t, t^{\prime}\right)\right] \Gamma_{n}\left(t^{\prime}\right)\right\}
\end{aligned}
$$

Here, $\mathcal{G}_{n m}\left(t, t^{\prime}\right)=2 \operatorname{Re} \sum_{\lambda} \chi_{\lambda}^{2}\left[(n+1) \delta_{m n+1}+\right.$ $\left.n \delta_{m n-1}\right] e^{i \varpi_{0}(m-n)\left(t-t^{\prime}\right)} R_{\lambda}\left(t-t^{\prime}\right) \mathcal{F}_{n m}\left(t, t^{\prime}\right)$ are the nonMarkovian transition probability densities that exhibit the stochastic behavior through the stochastic functionals $\mathcal{F}_{n m}\left(t, t^{\prime}\right)=\exp \left\{i \int_{t^{\prime}}^{t} d \tau\left[(m-n)\left(\omega(\tau)-\varpi_{0}\right)\right]\right\}$, while $R_{\lambda}(\tau)=N\left(\Omega_{\lambda}\right) e^{i \Omega_{\lambda} \tau}+\left[N\left(\Omega_{\lambda}\right)+1\right] e^{-i \Omega_{\lambda} \tau}$ correspond to the correlation functions for one-phonon transitions between the nearest vibrational levels with $N(\Omega)=$ $[\exp (\hbar \Omega / k T)-1]^{-1}$ being the Bose distribution function.

The main difficulty in solving the integro-differential equation (9) lies in a non-Markovian character of the respective integrands. Moreover, one has to make the explicit averaging of the stochastic functionals $\Gamma_{n}(t)$ and $\mathcal{F}_{n m}\left(t, t^{\prime}\right)$. However, when considering one-phonon transitions in the second order of perturbation theory in the relaxation interaction, the non-Markov property can be neglected [15]. Using this fact and taking into account that the characteristic time of stochastic processes $\tau_{\text {stoch }}$ is of the order of the thermal relaxation time $\tau_{T} \sim \tau_{\text {stoch }}$ and consequently $\tau_{\text {stoch }} \ll \tau_{\text {tr }}$, one can perform the stochastic averaging of (9) in the implicit form, which yields

$$
\begin{aligned}
& \frac{\partial}{\partial t} \gamma_{n}(t)=(n+1) W_{+} \gamma_{n+1}(t)+n W_{-} \gamma_{n-1}(t)- \\
& -\left[n W_{+}+(n+1) W_{-}\right] \gamma_{n}(t) .
\end{aligned}
$$

The respective averaged probabilities of transitions from the first to the zero $(+)$ and from the zero to the first $(-)$ vibrational levels are

$$
W_{ \pm}=2 \operatorname{Re} \sum_{\lambda} \xi_{\lambda} \varpi_{0} \Omega_{\lambda} \int_{0}^{\infty} d \tau e^{ \pm i \varpi_{0} \tau} R_{\lambda}(\tau) F(\tau),
$$

where $F(\tau)=\left\langle\left\langle\exp \left\{i \int_{0}^{\tau} d \tau^{\prime}\left[\omega\left(\tau^{\prime}\right)-\varpi_{0}\right]\right\}\right\rangle\right\rangle$ is the averaged correlation function of stochastic shifts.

As one can see, the general problem reduces to the problem of determining the model for levels' stochastic alternation $\omega(t)$, subsequent calculation of the correlation function $F(\tau)$, and final evaluation of transition 
probabilities $W_{ \pm}$. Note that, in the case of thermal excitation, initially only one energy level (zero) is occupied: $\gamma_{n}(0)=\delta_{n 0}$. Thus, the evolution of both the mean vibrational energy $\left\langle E_{\mathrm{vib}}(t)\right\rangle \equiv \sum_{n} \hbar \varpi_{0} \gamma_{n}(t)(n+1 / 2)$ and state occupancies $\gamma_{n}(t)$ are determined by the wellknown expressions $[4,5]$

$$
\left\{\begin{array}{l}
\left\langle E_{\mathrm{vib}}(t)\right\rangle=\hbar \varpi_{0}\left[N\left(\varpi_{0}\right)\left(1-e^{-\kappa t}\right)+1 / 2\right], \\
\gamma_{n}(t)=\frac{\left(e^{\hbar \varpi_{0} / k T}-1\right)\left(1-e^{-\kappa t}\right)^{n}}{\left(e^{\hbar \varpi_{0} / k T}-e^{-\kappa t}\right)^{n+1}}
\end{array}\right.
$$

In the above equations, $\kappa \equiv W_{+}-W_{-}$is the effective rate constant of thermal excitation which fully describes the kinetics of relaxation processes. Therefore, the quantity $\kappa$ represents a major kinetic parameter of interest which has been sought as a general solution of the problem considered in the one-phonon approximation. It should be noted that the state occupancies could also be represented in the equivalent form $\gamma_{n}(t)=\left[\left\langle\gamma_{n}(t)\right\rangle-1 / 2\right]^{n}\left[\left\langle\gamma_{n}(t)\right\rangle+1 / 2\right]^{-n-1}$, where $\left\langle\gamma_{n}(t)\right\rangle \equiv N\left(\varpi_{0}\right)\left(1-e^{-\kappa t}\right)+1 / 2$ is the average mean occupancy over vibrational states.

\section{Rate Constant}

Let us use a symmetric dichotomous process $\alpha(t)$ as a model for stochastic shifts of the molecular frequency $\omega(t)(3)$ from its mean value $\varpi_{0}$. During such a process, the frequency exhibits jumps between two equiprobable values $\omega(t)-\varpi_{0} \equiv \alpha(t)= \pm \sigma$ at random times with mean frequency $\nu$. It can be shown [15] that, in this case, $F(\tau)=\left(k_{1} e^{-k_{2} \tau}-k_{2} e^{-k_{1} \tau}\right) /\left(k_{1}-k_{2}\right)$ in (11), where $k_{1,2}=\frac{1}{2}\left(\nu \pm \sqrt{\nu^{2}-4 \sigma^{2}}\right)$. Then, according to (12), the rate constant can be represented in the following general form:

$$
\begin{aligned}
& \kappa=2 \varpi_{0} \sigma^{2} \nu \sum_{\lambda} \xi_{\lambda} \Omega_{\lambda} \times \\
& \times\left\{\frac{1}{\left[\left(\Omega_{\lambda}-\varpi_{0}\right)^{2}-\sigma^{2}\right]^{2}+\nu^{2}\left(\Omega_{\lambda}-\varpi_{0}\right)^{2}}-\right. \\
& \left.-\frac{1}{\left[\left(\Omega_{\lambda}+\varpi_{0}\right)^{2}-\sigma^{2}\right]^{2}+\nu^{2}\left(\Omega_{\lambda}+\varpi_{0}\right)^{2}}\right\} .
\end{aligned}
$$

From (13), one can see that the rate depends on the dynamic $\left(\omega_{0}, \Omega_{\lambda}, \xi_{\lambda}\right)$, shifted $\left(\varpi_{0}=\omega_{0}+\sigma\right)$, and stochastic $(\sigma, \nu)$ parameters of the system. The last additionally provides for a temperature dependence of the rate, as
$\sigma=\sigma(T)$ and $\nu=\nu(T)$. Therefore, to perform the further analysis, one has to represent the rate constant in the convenient form.

First of all, let us make summation over the normal modes $\lambda$ in (13). Let the corresponding factor of nonadiabaticity, which characterizes the constant of intermolecular anharmonicity (6), have a structure

$\xi_{\lambda}=2 \Omega_{\lambda} I\left(\Omega_{\lambda}\right)$,

where the function $I\left(\Omega_{\lambda}\right)=\eta /\left(\Omega_{\lambda}^{2}+\eta^{2}\right)$ determines the density of a spectral distribution of frequencies $\Omega_{\lambda}$. In this distribution, the width parameter $\eta$ plays a role of adiabatic width for heat bath phonon spectra (in (14), the adiabatic limit $\xi_{\lambda} \rightarrow 1$ arrives at $\Omega_{\lambda} \rightarrow \eta$ ). The specific value of $\eta$ depends on the model chosen for the adiabatic interaction in a given system. If vibrations in a molecule and the medium merge into the unified set of vibrational states, then the heat bath with an infinite set of normal modes forming an almost continuous spectrum can be chosen as a model of molecular environment. The continuity of such a spectrum means that, after the transformation of a sum over $\lambda$ into an integral over $\Omega$ and its subsequent calculation, the value of $\eta$ must be turned to zero: $\eta \rightarrow+0$.

This approximation is usually adequate in homogeneous structures of a similar molecular nature, for example, in crystals. Another situation could occur in heterogeneous systems (disordered and amorphous structures, biological macromolecules, etc.), especially at finite temperatures. In such systems, the adiabatic spectral widths $\eta=\eta_{\lambda}$ would have an additional dependence on the distribution density of normal modes $\lambda$. In particular, their values might coincide with frequencies of certain adiabatic (mechanical) modes $\eta_{\lambda} \simeq \Omega_{\lambda}$ and would be much smaller $\left(\eta_{\lambda} \ll \sigma\right)$ than the standard deviation for thermal fluctuations $\sigma$. On the other hand, the values of $\eta_{\lambda}$ should considerably differ from those of nonadiabatic (optical) modes $\eta_{\lambda} \ll \sigma \ll \Omega_{\lambda}$. But in all cases, the shifted frequency of molecular vibrations $\varpi_{0}=\omega_{0}+\sigma$, which linearly rises with the mean random field $\sigma$, significantly exceeds the adiabatic width: $\varpi_{0} \gg \eta_{\lambda}$. Thus, in the one-phonon approximation $\Omega_{\lambda} \simeq \varpi_{0}$, the values of $\eta_{\lambda}$ must be considered small for all $\lambda$.

Note that, in the case of a uniform distribution of normal modes, a width of adiabaticity $\eta$ forms physically, in fact, the finest scale over the frequency axis $\Omega_{\lambda}$. This allows one to make a transformation in (13) and (14) to the variables $\Omega_{\lambda} \equiv(\Delta \Omega) \lambda=\eta \lambda$, where the elementary frequency shifts $\Delta \Omega \equiv \Omega_{\lambda+1}-\Omega_{\lambda}=\eta$ will not depend on the number of a vibrational mode $\lambda$. By so doing, one can sum over $\lambda$ in (13) up to infinity and, taking 
the continuity condition $\eta=d \Omega \rightarrow 0$ into account, make a transformation from a sum of functions $f\left(\Omega_{\lambda}\right)$ to the integral of a function $f(\Omega)$ by the rule $\sum_{\lambda=1}^{\infty} f\left(\Omega_{\lambda}\right)=$ $\lim _{\eta \rightarrow 0}\left[\frac{1}{2 \pi \eta} \int_{0}^{\infty} f(\Omega) d \Omega\right]$. As a result, with regard for (14), elementary calculations yield

$\kappa=\frac{4}{\pi}\left(\omega_{0}+\sigma\right) \int_{0}^{1+\frac{\omega_{0}}{\sigma}} d z \frac{\nu}{\sigma}\left[\left(1-z^{2}\right)^{2}+\frac{\nu^{2}}{\sigma^{2}} z^{2}\right]^{-1}$.

We see that, since sum (13) does not generally depend on the Bose distribution function for molecular vibrations $N\left(\varpi_{0}\right)$, the very knowledge of the particular nonadiabaticity parameter $\xi_{\lambda}$ (14) allows us to reduce the rather complicated equation (13) to the simple integral (15) that only contains the unknown dimensionless stochastic factors $z=\Omega / \sigma, \nu / \sigma$, and $\varpi_{0} / \sigma$. As the second step, let us explore a specific model for the temperature dependence of stochastic parameters. For this purpose, we use the thermodynamic approach that was originally proposed in $[15,16]$. In this approach, the standard deviation of the amplitude of a dichotomous process $\sigma$ is linked to the mean square of the energy fluctuation $\overline{\delta E^{2}}$ via the relation $\pi^{2} \hbar^{2} \sigma^{2}=\overline{\delta E^{2}}$. On the other hand, from the theory of thermodynamic fluctuations in canonical ensembles, we know that the latter quantity is given by $\overline{\delta E^{2}}=k T^{2}(\partial \bar{E} / \partial T)$, where $\bar{E}=2 \pi \hbar \nu$ is the mean energy of dichotomous fluctuations with frequency $\nu$. In the classical limit, the mean energy $\bar{E}=\hbar \omega_{c}\left[N\left(\omega_{c}\right)+1 / 2\right]$ per a separate degree of freedom must turn out to the thermal energy $k T$, while in the quantum limit, the same quantity must correspond to the energy of zero-point oscillations with some correlation frequency $\omega_{c}$. Therefore, independently of the molecular system under study, the stochastic fluctuation parameters can be determined by the following expressions:

$\left\{\begin{array}{l}\sigma=\left(\omega_{c} / \pi\right) \sqrt{N\left(\omega_{c}\right)\left[N\left(\omega_{c}\right)+1\right]} \\ \nu=\left(\omega_{c} / 2 \pi\right)\left[N\left(\omega_{c}\right)+1 / 2\right]\end{array}\right.$

The general relations (15) and (16) allow one to examine important limiting cases. Since a characteristic scale for stochastic values (16) is determined by a frequency $\omega_{c}$, in the case of sufficiently low temperatures $\left(\hbar \omega_{c} \gg\right.$ $k T$ ), the upper limit of integral (15) can be turned to infinity. In this case, $\int_{0}^{\infty} d z \frac{\nu}{\sigma}\left[\left(1-z^{2}\right)^{2}+\frac{\nu^{2}}{\sigma^{2}} z^{2}\right]^{-1}=\pi / 2$, and

$\kappa=2\left(\omega_{0}+\sigma\right)=2 \omega_{0}+\frac{2 \omega_{c}}{\pi} e^{\hbar \omega_{c} / 2 k T}\left[e^{\hbar \omega_{c} / k T}-1\right]^{-1}$.
Conversely, at sufficiently high temperatures $\left(\hbar \omega_{c} \ll\right.$ $k T)$, the numerical calculation of the integral in (15) yields

$\kappa=\frac{2}{\pi}\left((A+4) \omega_{0}+A \sigma\right)+O\left(\frac{\omega_{0}}{\sigma}\right)$,

where $A \simeq 2.10357$. Note, that the value of the integral in (15) rapidly tends to $\pi / 2$, as $\hbar \omega_{0} / k T$ increases. It is equal to $\pi / 2$ up to the fourth order even when $\hbar \omega_{0} / k T=$ 1. Therefore, practically for all temperatures of interest, the corresponding thermal excitation rate constant $\kappa$ can be well described by the quite simple analytic relation (17).

It is worth noting that Eq. (15) can be proved only by certain approximations that simplify the general character of the thermal excitation. Particularly, we use both the model of one-phonon transitions between the nearest energy levels and the dichotomous mechanism of frequency alternation due to the interaction with random motions in the environment.

The first approximation is a direct consequence of the bilinearity of the intermolecular anharmonicity operator over mutual deviations of the nuclei of a molecule under consideration and molecules in the environment from equilibrium positions (5). As is known, the account of nonlinear terms significantly complicates the problem by making a relaxation of the system essentially multiphononic. In particular, transitions over the one vibrational level become possible, as well as transitions accompanied by the simultaneous creation or annihilation of several phonons in the medium $[5,6]$. However, the consideration of such effects is important only at very high temperatures, when the vibrational structure of a molecule becomes undefined in the quantum sense. This is also the case for molecules with internal anharmonicity, when only the lowest vibrational levels are well defined, while the energetically higher levels merge into a continuous spectrum.

The second approximation is not critical. Thus, using a common Gaussian model in (11) with the stochastic parameters $\sigma$ and $\nu$ analogous in their meaning instead of dichotomous model, one obtains the rate constant in the form

$\kappa=\frac{4}{\pi}\left(\omega_{0}+\sigma\right) \int_{0}^{1+\omega_{0} / \sigma} d z \frac{\nu}{\sigma}\left[1+\frac{\nu^{2}}{\sigma^{2}} z^{2}\right]^{-1}$.

This equation can be exactly reduced to the same form (17) as in the low temperature limit of dichotomous model (15) due to the $\delta$-like character of its integrand. 
In general, the presence of two terms in expressions (15), (17), and (18) for the rate constant $\kappa$ physically means that, in the harmonic (one-phonon) approximation, the process of molecular thermal excitation is governed by the two additive mechanisms. The first is a quantum mechanism $\left(\sim 2 \omega_{0}\right)$ being essentially temperature-independent, while the second is a quasiclassical one $(\sim 2 \sigma)$ being activated with increase in the temperature. The mutual contribution of these mechanisms is determined by characteristic frequencies of vibrations in a molecule $\left(\omega_{0}\right)$ and the medium $\left(\omega_{c}\right)$ with relation to the thermal frequency $\omega_{T} \equiv k T / \hbar$. Thus, in the quantum limit $\omega_{0}>\omega_{c} \gg \omega_{T}$, the inequality $\omega_{0} \gg \sigma$ holds, and the main contribution to the thermal excitation is introduced by quantum transitions between the nearest vibrational levels of a molecule. In this case, $\kappa=2 E_{0} / \hbar(17)$, which corresponds to the rate speed limit for quantum transitions between orthogonal states with energy dispersion $E_{0}=\hbar \omega_{0}[17,18]$ in a full accordance with the uncertainty principle. In the limit of classical transitions, $\omega_{c}<\omega_{0} \ll \sigma<\omega_{T}$, and $\kappa=2 \omega_{T} / \pi=2 k T / \pi \hbar$, which coincides with the Einstein relation for the adiabatic rate of Brownian oscillator relaxation $[13,14]$. In this case, if the condition $\omega_{c}<\omega_{0}$ holds, then there are no transitions between the energy levels of a molecule according to the adiabatic theorem. As a result, the thermal excitation occurs via a simple increase of the shifted vibration frequency $\varpi_{0}=\omega_{0}+\sigma \approx \sigma=k T / \pi \hbar$ due to its direct coupling to random motions in the environment.

We have to note that the quantity $\kappa$ depends on the Planck constant $\hbar$ only through the characteristic time of thermal relaxation $\tau_{T}=\omega_{T}^{-1}=\hbar / k T$ involved in the Bose distribution function for correlation fluctuations in the medium $N\left(\omega_{c}\right)=\left[\exp \left(\omega_{c} \tau_{T}\right)-1\right]^{-1}$. This property allows one to make a safe transformation from the quantum $(T \rightarrow 0)$ to quasiclassical $(\hbar \rightarrow 0)$ limit in the heat bath, by considering the processes of thermal relaxation to be very fast $\omega_{T} \gg \omega_{0}>\omega_{c}$ $\left(\tau_{T} \rightarrow 0\right)$ or setting the temperature relatively high $T \rightarrow \infty$. By turn, the corresponding transformation from the temperature-independent asymptotics to that linear in the temperature for $\kappa$ is well reproduced in the unified manner. Therefore, one needs neither knowledge of the molecular $\left(\omega_{0}\right)$ or correlation $\left(\omega_{c}\right)$ vibrational frequencies, nor any introduction of additional factors for a "correction" of the temperature dependence of the rate constant in the classical limit [11]. This characteristic feature solves the so-called harmonic oscillator paradox [19]. Indeed, one may think that, in accordance with quantum theory, the overall probability of transitions between the nearest levels of an oscillator, for example between the $n$-th level and the $(n-1)$ th one, should be proportional to the total width of these levels (i.e., to the factor $\hbar^{2} \chi_{\lambda}^{2}(2 n-1)(2|n\rangle\langle n|+1)$ ) (5), (11). But while turning to the classical limit $n \rightarrow \infty$, which is achieved at high temperatures (when $\omega_{0} \ll k T / \hbar \rightarrow \infty$ or $\left.N\left(\omega_{0}\right) \sim\langle\mid n\rangle\langle n \mid\rangle \rightarrow \infty\right)$, this factor diverges. Thus, the concept of a classical harmonic oscillator as a dynamic system being at the extremely high temperature and having an infinite set of densely distributed levels makes no sense. However, it follows from the general equations (12) that the evolution of both the average vibrational energy and oscillator state occupancies at the thermal excitation depends on the rate constant $\kappa$ that is determined not by the sum, but by the difference between level widths of the first and zero levels at any temperature and any level distribution density, rather than between those of the $n$th and $(n-1)$ th levels. Therefore, both the numbers of vibrational levels and the respective Bose functions for molecular vibrations $N\left(\omega_{0}\right)$ remove from explicit consideration introducing any contribution to the value of rate constant $\kappa(13)$, that limits the process of establishing a final thermal equilibrium in the molecule.

1. G.V. Hartland, Chem. Sci. 1, 303 (2010).

2. A. Gaiduk, P.V. Ruijgrok, M. Yorulmaz, and M. Orrit, Chem. Sci. 1, 343 (2010).

3. P. Zijlstra, J.W.M. Chon, and Min Gu, Nature 459, 410 (2009).

4. A. Nitzan and R. Silbey, J. Chem. Phys. 60, 4070 (1974).

5. E.G. Petrov and V.I. Teslenko, Theor. Math. Phys. 38, 128 (1979).

6. V.I. Teslenko, Theor. Math. Phys. 81, 203 (1989).

7. V.I. Teslenko, Ukr. J. Phys. 34, 1588 (1989).

8. E.G. Petrov and V.I. Teslenko, Ukr. J. Phys. 35, 1106 (1990).

9. T. Kato and Y. Tanimura, J. Chem. Phys. 117, 6221 (2002).

10. Q. Shi and E. Geva, J. Chem. Phys. 118, 7562 (2003).

11. H. Fujisaki, L.Bu, and J.E. Straub, Adv. Chem. Phys. 130, B, 179 (2005).

12. D.M. Leitner, Adv. Chem. Phys. 130, B, 205 (2005). 
13. Y. Yan and R. Хu, Annu. Rev. Phys. Chem. 56, 187 КІНЕТИКА ТЕПЛОВОГО ЗБУДЖЕННЯ МОЛЕКУЛИ (2005). У КОНДЕНСОВАНОМУ СЕРЕДОВИЩ

14. I. Goychuk and P. Hanggi, Adv. Phys. 54, 525 (2005).

В.І. Тесленко, Д.Ю. Яченко

15. E.G. Petrov and V.I. Teslenko, Chem. Phys. 375, 243 (2010)

16. V.I. Teslenko, E.G. Petrov, A. Verkhratsky, and O.A. Krishtal, Phys. Rev. Lett. 104, 178105 (2010).

17. L.I. Mandelshtam and I.E. Tamm, J. Phys. (U.S.S.R.) 9, $122(1945)$.

18. D.C. Brody, J. Phys. A: Math. Gen. 36, 5587 (2003).

19. B.Y. Zeldovich, A.M. Perelomov, and V.S. Popov, J. Exp. Theor. Phys. 2(8), 589 (1968).

Received 01.03.11

Р ез ю м е

Побудовано мікроскопічну модель теплового збудження основного коливального стану молекули, що контактує з конденсованим середовищем. Отримано керуюче рівняння для еволюції заселеностей коливних рівнів. В аналітичному вигляді визначено константу швидкості, яка лімітуе процес теплового збудження молекули. Показано, що при низьких температурах ця величина не залежить від температури, збігаючись за принципом невизначеності з верхньою границею для швидкості квантових переходів, а при високих температурах - лінійно росте з температурою згідно із співвідношенням Ейнштейна для швидкості адіабатичних переходів. 